D.T. Wickramasinghe, L. Ferrario, and G.V. Bicknell, eds.

\title{
Disk-Corona Models and X-Ray Emission from Seyfert Galaxies
}

\author{
Laura Maraschi \\ Osservatorio Astronomico di Brera, via Brera 28, 20121 Milano, Italy \\ Francesco Haardt \\ Department of Astronomy 83 Astrophysics, Institute of Theoretical \\ Physics, Göteborg University \& Chalmers University of Technology, 412 \\ 96 Göteborg, Sweden
}

\begin{abstract}
The current status of understanding of the X-ray emission from Seyfert galaxies involves Comptonization of soft photons by hot subrelativistic electrons. After briefly reviewing the early theoretical basis for the presence of hot optically thin plasma in or around accretion disks and the key observations that led to develop the presently popular model of an accretion disk with a hot corona, we summarize recent progress in accretion models that take into account energy dissipation and/or angular momentum transport in the corona. Finally, adopting the simple scheme of a homogeneous plane parallel corona, we discuss in detail the dependence of the X-ray spectrum on the coronal parameters. Despite the strong coupling between optical depth and temperature which determines a spectral shape insensitive to their precise values, moderate spectral changes are possible. The spectral variability patterns can be used as diagnostics for coronal physics and should allow to determine whether the optical depth of the corona is dominated by $e^{+} e^{-}$pairs.
\end{abstract}

\section{Introduction}

The main uncertainty in the thory of accretion disks is on the physical processes responsible for energy dissipation and angular momentum transport. Despite important progresses in understanding turbulent and magnetic viscosity it is still impossible to anticipate the precise mechanisms at work in astrophysical plasmas, in particular in accretion disks. The emitted spectra are not too sensitive to these mechanisms if the disk is optically thick and the dissipation occurs within the thick layer. However the high energy spectra emitted by black hole binaries and active galactic nuclei indicate conditions far from thermodynamic equilibrium, implying that the above conditions are strongly violated in the inner regions of highly luminous accretion disks around black holes.

We review here shortly the evolution of accretion models involving a hot corona as an important site of energy dissipation and the observational grounds for such picture in the case of Seyfert 1 galaxies. We describe the basic features of the radiative coupling and feed back effects of a sandwich configuration that can 
account for the observed X-ray spectra and present new results on the spectral "dynamics" expected in such models.

\section{Early Ideas about X-ray Emission from Accretion Disks}

The importance of Comptonization for the high energy spectra of luminous accretion disks was recognized since the seminal paper by Shakura and Syunyaev (1973) and it is a pleasure to recall that both authors participated to this conference. However the high temperature and power law spectral shape observed for Cyg X-1 and the instability problems of radiation pressure supported and optically thin disks challenged the selfconsistency of the "standard" model leading Shapiro, Lightman \& Eardley (1976) to hypothesize that pressure support could be due to hot ions, weakly coupled to the radiating electrons in a "two temperature" optically thin plasma. Applying the model to Cyg X-1 these authors noted the desirability of having the comptonization parameter $y=\tau(\Delta \epsilon / \epsilon)$ close to one, without however finding a motivation for such value within the model itself ( $\tau$ is the optical depth of the corona and $(\Delta \epsilon / \epsilon)$ the average energy gain per scattering).

The possibility of a hot corona above a relatively cool accretion disk was discussed by Liang and Price (1977) and subsequently Liang (1979) first noted that a sandwich configuration would

1. be "natural" in a magnetoturbulent disk since dissipation of magnetic energy occurs preferentially in a rarefied atmosphere,

2. solve the stability problem (see Ionson \& Kuperus 1984),

3. imply a feedback between disk and corona that would determine a value of $y$ close to 1 .

The physics of magnetic dissipation in accretion disks was substantiated and applied to galactic black hole candidates by Galeev, Rosner \& Vaiana (1979), while a basic treatment of Comptonization was given by Sunyaev \& Titarchuck (1980).

The relevance of Comptonization for AGN spectra was first discussed by Katz (1976) and later by Lightman Giacconi and Tananbaum (1978) for the $\mathrm{X}$-ray spectrum of NGC 4151 . The latter authors first noted that the inferred parameters for NGC 4151 implied high optical depth for pair production suggesting a cut off in the observed spectrum at $500 \mathrm{keV}$ due to this process.

Non thermal pair plasmas were studied in depth in the 80 's in relation to the X-ray emission of AGN. We will not try to summarize this subject here but refer to Svensson (1994) for a comprehensive review.

\section{Key Observations}

Different "types" of observations were instrumental in leading to the present widely adopted scheme of a cool optically thick disk embedded in a hot corona. New observations in the medium-hard X-ray range with the GINGA satellite, 
revealed the signature of "cold matter" in the X-ray spectra of Seyfert galaxies, detecting the $K_{\alpha}$ emission line of Fe and a broad hump around $10 \mathrm{keV}$ as expected for "reflected" X-rays (Pounds et al. 1990).

Systematic comparisons of the UV and X-ray emission in quasars and Seyfert galaxies (Sanders et al. 1989, Walter and Fink 1993) indicated that the power emitted in the latter was at most comparable to but not larger than that emitted in the UV. In order to have similar accretion power, the two emitting regions must be at a similar distance from the black hole and a configuration with a hot inner region surrounded by a larger cooler disk is problematic.

A possible way out of this difficulty is provided by the new class of advection dominated accretion disk solutions (see the review by Narayan at this meeting) in which the radiative efficiency of the inner disk is reduced. In these solutions however, as in the earlier model by Shapiro Lightman \& Eardley the value of the Comptonization parameter is determined "a posterior".

Moreover observations of correlated variability in the UV and X-rays (NGC 4151, Perola et al., 1986, and NGC 5548, Clavel et al., 1992) suggested that a large fraction of the UV emission is due to reprocessing of X-rays, implying that the UV emitting region intercepts a large fraction of the X-rays .

The most natural configuration accounting for the above points is that of a two phase or sandwich disk model as described below. An important prediction of this scheme was that a thermal cut-off should be expected above $100 \mathrm{keV}$ in the X-ray spectra of Seyfert nuclei. The OSSE experiment on the Compton Gamma Ray Observatory indeed confirmed this prediction, showing a substantial steepening of the spectra in the hard X-ray range (e.g. Maisack et al. 1993, Zdziarski et al. 1995). These results set strong limits to the possible role of non-thermal pair plasmas and provide a measurement of the total luminosity in the comptonized component (due to the "convergence" of the integral flux) which confirms that the luminosity in the UV is comparable or larger than in $\mathrm{X}$-rays.

\section{A Homogeneous Two Phase Model for the X-ray Emission}

Guided by the above theoretical and observational points we analized the radiative transfer and coupling in the simple scheme of a sandwich model with a cool optically thick layer embedded in a hot optically thin one. Using a plane parallel limit a thermal balance equation can be written for each of the two "phases" assuming that a fraction $f$ of the gravitational power is dissipated in the hot corona and a fraction $1-f$ in the optically thick disk. The coupling between the two "phases" arises from the fact that the Comptonization process in the hot corona gives rise to quasi-isotropic X-ray radiation. Therefore, in the plane parallel limit, only half of it escapes from the source while the other half impinges on the cool disk. The latter is in part $(10-20 \%)$ reflected, giving rise to the observed spectral hump in the $10-30 \mathrm{keV}$ range; in small part it is reemitted as an Fe fluorescence line, but the largest part $(80-90 \%)$ is absorbed, reprocessed and reemitted into black-body photons which contribute to the soft photon input for Comptonization.

The two coupled equations determine the value of the Compton amplification parameter $A=1+L_{C o m p} / L_{\text {soft }}$, necessary to close the loop (Haardt \& 
Maraschi 1991, hereinafter HM91). $A$ is determined by the only free parameter $f$ and for small f $A \simeq 1+f$, while for $f=1 A \simeq 3$. The exact value of $A$ in the limit $f=1$ depends on the precise value of the albedo and on the degree of anisotropy of the comptonized radiation and is very important because it fixes the spectral shape of the comptonized X-ray radiation. In the theory of Comptonization the spectral index depends on the temperature $T_{C}$ and optical depth of the comptonizing electrons. Therefore fixing $A$ implies a fixed spectral shape and a relation between $T_{C}$ and $\tau$.

If the corona is dominated by electron positron pairs its optical depth is determined by the compactness parameter, defined as $l_{c}=\frac{\sigma_{T}}{m_{e} c^{2}} H / R^{2} L$ where $\mathrm{H}$ is the scale height of the corona, $R$ the radius of the underlying disk and $L$ the total luminosity of the system. The relation between $T_{C}$ and $\tau$ imposed by the feed back effect transforms then into a relation between $T_{C}$ and $l_{c}$, with higher values of the compactness corresponding to lower values of the temperature (HM91).

From the approximate estimates in HM91 it was clear that in order to reproduce the average X-ray spectrum of Seyfert galaxies it was necessary to have $f \simeq 1$, which implied that practically all the energy was dissipated in the corona and the cool disk acted only as a "passive" reprocessor. With this assumption, the model could account for the average value and for the relatively small dispersion of spectral indices of Seyfert I galaxies (Nandra \& Pounds 1994), for the reflection hump and for the Fe emission line. It predicted a spectral cut off in the hard X-ray range which was revealed by OSSE (see above).

Improved treatment of radiative transfer and detailed spectral calculations (Haardt, 1993; Haardt and Maraschi 1993; Titarchuk 1994) confirmed and refined these results. More accurate solutions for the pair corona model are discussed in Poutanen and Svensson (1996) and Stern et al. (1995), not only for a slab but also for cylindrical and hemispherical geometry for the active region.

\section{Accretion models of disk+corona systems}

The success of the two phase model in explaining the X-ray properties of Seyfert galaxies and the realization that most of the accretion power should be released directly in the corona, presumably through magnetic buoyancy and reconnection above the optically thick disk, motivated new investigations of this configuration with different assumptions as to the share of angular momentum tranfer, energy dissipation and accretion rate and with different boundary conditions between disk and corona.

Nakamura \& Osaki (1993) describe the two phases as two $\alpha$ disks with accretion taking place in the cool disk only. However, as additional constraint the average coronal pressure $P_{c}$ is taken to be equal to the average disk pressure $P_{d}$, clearly an unphysical assumption. A similar approach is adopted by Kusunose $\&$ Mineshige (1994) who assume that mass accretion and energy dissipation are shared between disk and corona again with the unphysical condition $P_{c}=P_{d}$.

Svensson \& Zdziarski (1994) model the cool phase as an $\alpha$ disk except for assuming that a fraction $f$ of the power release associated with the angular momentum transfer takes place above an optical depth $\tau_{c}$ which is treated as a free parameter due to the poor knowledge of the microphysical processes. 
They show that in the case of a strong corona (large $f$ ) the unstable radiation dominated region disappears (see also Ionson and Kuperus, 1984 and Chen 1995) coronal pressure and irradiation only affects the surface layers of the cold disk.

A more ambitious approach is that of Zycki et al. (1995) and Witt, Czerny and Zycky (1996) who aim at determining the fraction of power dissipated in the corona selfconsistently rather than treating it as a free parameter. They describe the disk and corona as accretion disks with different $\alpha$ parameters, imposing pressure balance at the base of the corona, and allowing the fraction of mass accretion through the disk and corona to be a function of radius.

In the first paper they find that coronal solutions are not allowed at high accretion rates due to the impossibility of satisfying the hydrostatic equilibrium equation, while in the second they include vertical supersonic outflow. In the latter case the corona forms only for accretion rates larger than a limiting value and the fraction of energy dissipated in the corona decreases with increasing accretion rate. The interest of this result is clear in view of the possible comparison with observational results for black hole binaries in different intensity states or for a comparison of the average properties of Seyfert galaxies and quasars. Although a large uncertainty still exists in the applicability of an $\alpha$ description of viscosity, especially in this complex case, this approach is very promising.

\section{The Two-Phase Model Revisited: A Patchy Corona?}

The simple two phase model originally proposed has two strong implications. First, the luminosity in the soft blackbody photons emitted by the surface underlying the comptonizing corona must be equal to the $\mathrm{X}$-ray luminosity (neglecting factors of a few due to the different angular dpendences of the two components). Moreover their intensities must be "perfectly" correlated in time. If we identify the blackbody component with the total UV emission, both these predictions are violated. In fact, as mentioned earlier (see section 3), if the spectral steepening in the hard X-ray range measured by OSSE for a few Seyfert galaxies is a general property, the estimated "bolometric correction" for the X-ray luminosity yields values smaller than the UV luminosity (Walter and Fink 1993). At the same time the correlation between UV and X-ray luminosity is present on timescales of days but may not hold on shorter timescales.

Both these difficulties can be solved in a more realistic coronal model, as proposed by Galeev Rosner \& Vaiana (1979), where the energy stored in the magnetic field is released in active regions covering only part of the cool disk (Haardt, Maraschi \& Ghisellini 1994). The feed back and X-ray spectral properties discussed earlier can be maintained if the power reprocessed under the active regions dominates that which is locally dissipated by other processes. However the disk emission not covered by active regions can leak out freely. The condition that $f=1$ is therefore not necessary on the large scale.

The active regions should produce hot spots on the disk giving rise to a rapidly varying component contributing in part to the UV bump and in part to the so called "soft excess" observed in many Seyferts (e.g. Mushotzky, Done \& Pounds, 1993). Also the flat limit may not be valid for each region allowing some more freedom in the expected spectral shapes (see for a review Svensson 1996) 


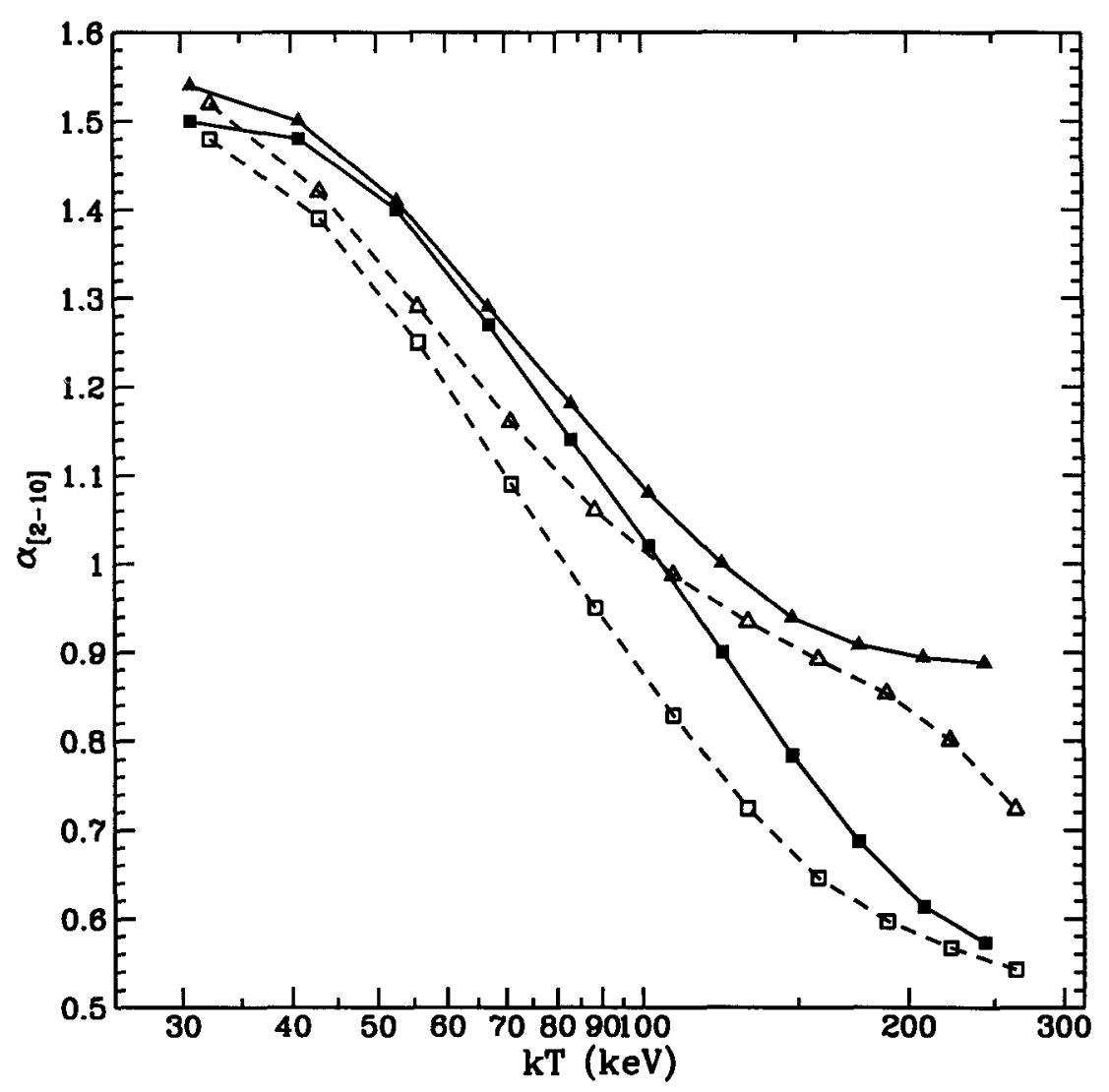

Figure 1. The total (direct power law + Comptonized reflection) spectral index in the $[2-10] \mathrm{keV}$ range $\alpha_{[2-10]}$ is plotted against the coronal electron temperature $k T$, for different values of the viewing angle and for different temperatures of the soft blackbody photons. Square symbols refer to a face-on line of sight, triangles to an inclination of $60^{\circ}$. Open symbols, connected with dashed lines, refer to a soft photon temperature $k T_{B B}=100 \mathrm{eV}$. Closed symbols connecetd with solid lines are for $k T_{B B}=50 \mathrm{eV}$. The curves show that harder spectra are expected for larger temperatures (lower optical depth) of the comptonizing electrons. The difference between results computed for different inclination angles is larger at higher temperatures (lower optical depths) due to the larger anisotropy of the first scattering. 


\section{Diagnostics of Coronal Models: Broad Band X-ray Variability}

In view of some existing and many more expected data on the spectral variability of Seyfert galaxies from the soft to the hard X-ray band we explored in some detail the behaviours expected in the model (Haardt Maraschi \& Ghisellini 1996). In fact, even adopting a fixed geometry for the active region(s) and despite an almost constant Compton parameter imposed by the feed back effect, ( $L_{C} / L_{S}=2$ for the slab geometry adopted in the following) the spectral shape in the medium X-ray range (characterized by a spectral index $\alpha_{[2-10]}$ ) depends somewhat on the parameters of the system (i.e. $\tau, T_{C}$ and $T_{B B}$ ). Moreover, the optical depth may depend on luminosity as well as the temperature of the soft reprocessed photons. Since $T_{B B}, \alpha_{[2-10]}$ and $T_{C}$ can be simultaneosly observed by broad band satellites like XTE or SAX or by the joint use of different satellites, it is interesting to compute the relations between these quantities. The most important results are summarized below.

The relation between the spectral index of the total spectrum $\alpha_{[2-10]}$ (including also Comptonization of the reflected spectrum ) and the temperature of the corona is shown in Figure 1, for different values of $T_{B B}$ and of the inclination angle. We recall that the optical depth does not appear explicitly but is related to the temperature by the balance equation for the corona (see also Pietrini and Krolik 1995). Values of $T_{C}$ in the range 30-300 keV, as suggested by observations (Maisak et al. 1993, Johnson et al. 1993, Madejski et al. 1995), correspond to optical depths between 1 and 0.1 .

The important points to note are the following:

1. Changes in $\tau$ and $T_{c}$ give rise to significant spectral variability despite the fact that the ratio $L_{C} / L_{S}$ is constant in the model. In the slab geometry considered here $\alpha_{[2-10]}$ is found to be in the range [0.4-1.4] for $\tau$ varying between 0.1 and 1 .

2. Steeper spectra are produced as $\tau$ increases approaching unity. Correspondingly, an increase of the spectral index is accompanied by a decrease of the coronal temperature, i.e. spectral index and coronal temperature are anticorrelated. The actual values of $\alpha$ and $T_{C}$ in Fig 2 refer to $L_{C} / L_{S} \simeq 2$, however the anticorrelation between spectral index and the electron temperature is a general feature common to models based on Compton cooling with fixed geometry. Note that the dispersion of the $\alpha_{[2-10]}$ vs. $k T$ relation is larger at higher temperature.

If the main contribution to $\tau$ comes from ordinary electrons, a reliable relation between luminosity and optical depth for the corona cannot be specified a priori. It is conceivable that changes in $\tau$ (e.g. fluctuations) occur at constant luminosity. This case is illustrated in Figure 2, where broad band spectra of constant total luminosity and different $\tau$ and $T_{C}$ are compared. The spectra pivot around $10-30 \mathrm{keV}$ : in the medium $\mathrm{X}$-ray range higher intensity corresponds to softer spectra. while in the hard $\mathrm{X}$-ray range larger intensity corresponds to harder spectra.

In contrast to the above situation, if the optical depth is due to pairs as expected in cases of high compactness, the luminosity (for fixed size) is a predictable and very strong function of $\tau$. This case is illustrated in Figure 3, where 


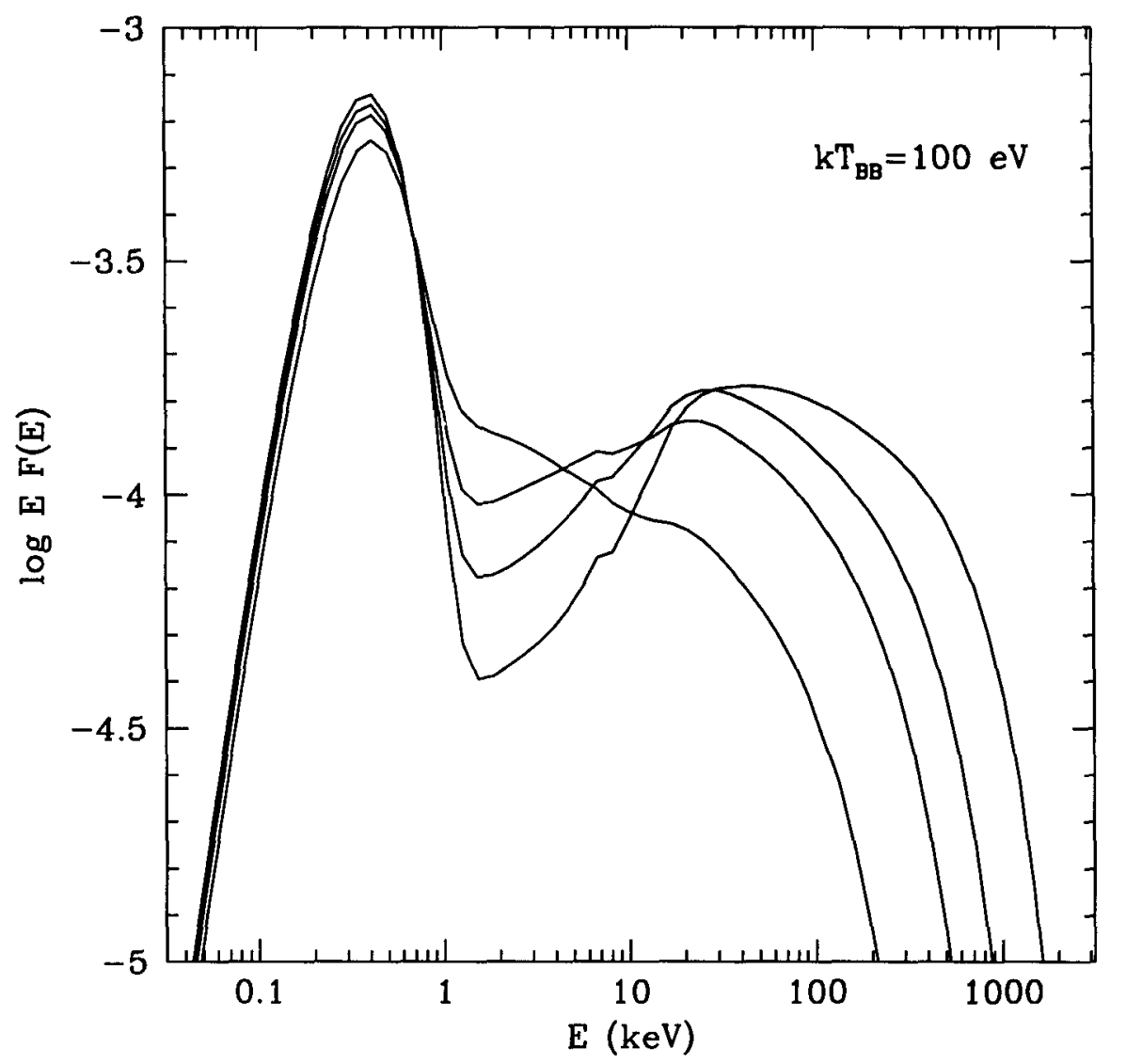

Figure 2. Comptonized spectra for constant luminosity and black body temperature $\left(k T_{B B}=100 \mathrm{eV}\right)$, computed for different $(\tau, \Theta)$ equlibrium values $[(0.63,011),(0.32,0.21),(0.2,0.3),(0.1,0.5)]$. A faceon line of sight is considered. 


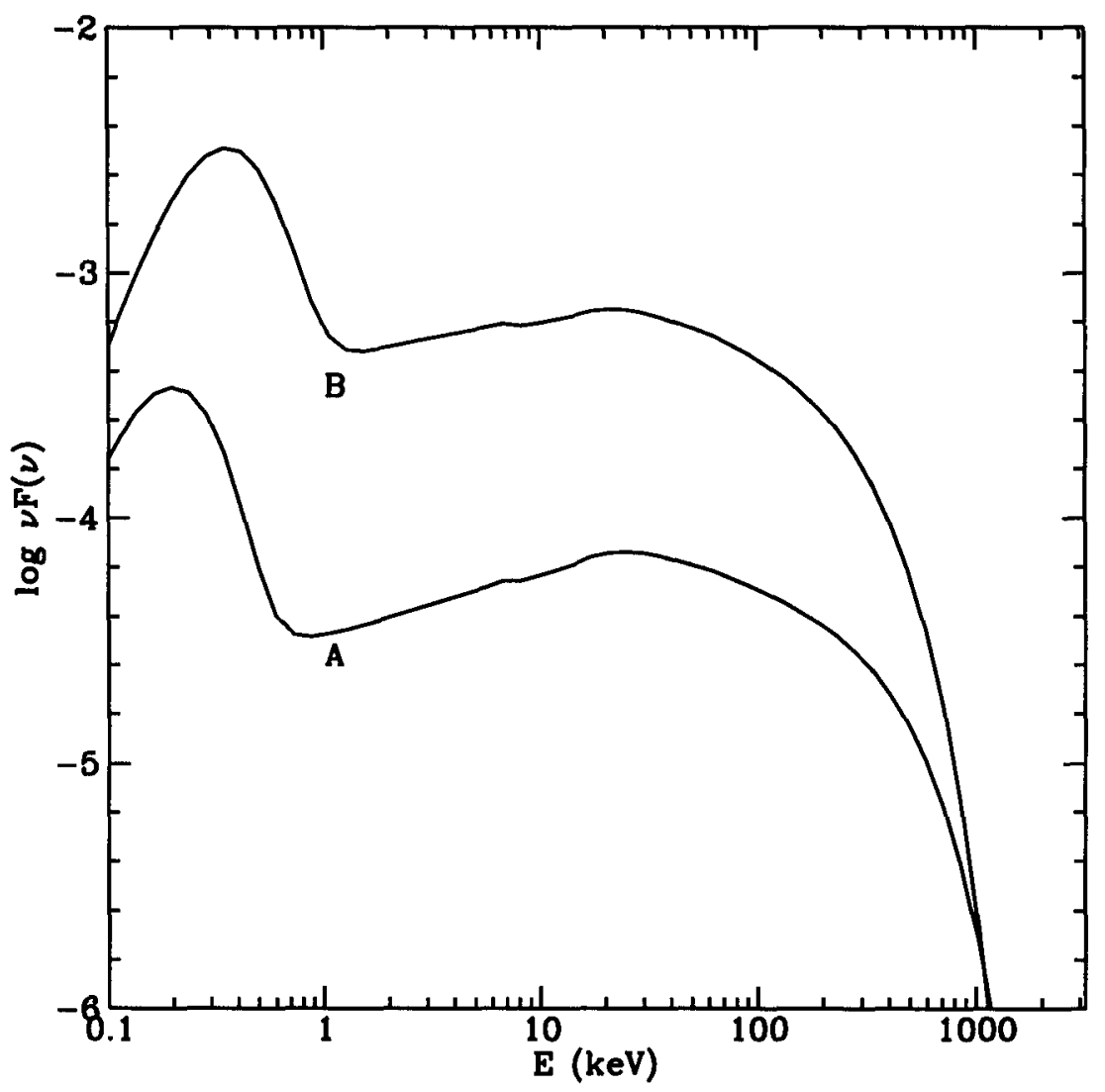

Figure 3. Spectra for $\ell_{c}=30(\mathrm{~A})$ and $\ell_{c}=300$ (B) are computed assuming a change of luminosity with constant emitting area. Spectrum " $\mathrm{A}$ " has $\tau=0.2, \Theta=0.3$ and $k T_{B B}=50 \mathrm{eV}$. Spectrum "B" has $\tau=0.32, \Theta=0.21$ and $k T_{B B}=89 \mathrm{eV}$. In both cases a face-on line of sight is considered. 


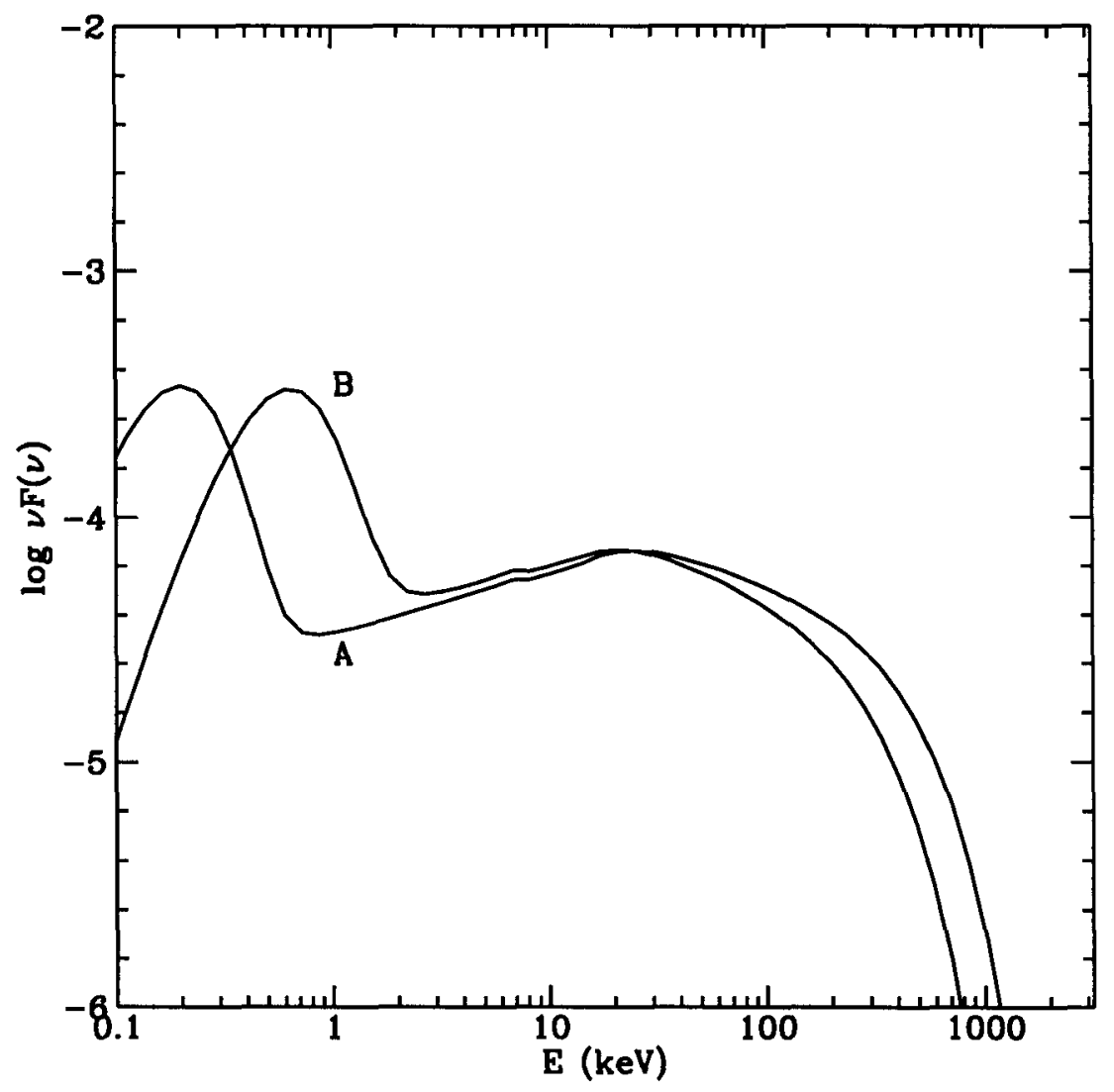

Figure 4. Same as Figure 3, but assuming a constant luminosity and a varying emitting area. As before, spectrum "A" $\left(\ell_{c} v=30\right)$ has $\tau=0.2, \Theta=0.3$ and $k T_{B B}=50 \mathrm{eV}$. Spectrum "B" (ell $\left.=300\right)$ has $\tau=0.32, \Theta=0.21$, but a much larger $k T_{B B}=158 \mathrm{eV}$. 
the two spectra refer to a change in luminosity by a factor 10 , but have very similar spectral shapes. The higher luminosity spectrum also has a consistently higher $T_{B B}$.

Finally in Figure 4 two spectra due to pair dominated coronae are compared, where the compactness was varied by changing the surface area of the active region at constant luminosity. Here a dramatic change in $T_{B B}$ is apparent with little spectral variations in the medium hard $\mathrm{X}$-ray range.

The two spectra also represent an extreme case of a large variation in the soft band without a correspondingly large variation at medium $\mathrm{X}$-ray energies. This demonstrates clearly that the correlation between $L_{c}$ and $L_{s}$ does not translate necessarily into a correlation of the observed medium and soft X-ray fluxes.

It seems quite premature to derive firm conclusions from the few broad band observations presently available. We note however that some spectral variability episodes observed in NGC 4151 (Perola et al. 1986), NGC 5548 (Done et al. 1995) and Mrk 766 (Leighly et al 1996), resemble more the pivoting behaviour illustrated in Figure 2 rather than those depicted in Figure 3 or 4, suggesting that these sources are not pair dominated.

\section{Conclusions}

Substantial progress in understanding emission processes from the inner regions of accretion disks has been achieved. X-ray observations suggest a two phase model, with hot optically thin gas coupled to a cooler optically thick layer, as may be expected for an accretion disk with large energy dissipation in a hot corona. However, the formation, heating and structure of the corona, its dependence on fundamental parameters like the accretion rate and central black hole mass are still largely unknown.

Broad band measurements from the soft to the hard X-ray band, possible with present satellites like ROSAT, ASCA, XTE and SAX offer the exciting perspective of investigating how the coronal parameters vary in time for the same source or differ statistically in different classes of sources. Important questions such as: is the optical depth dominated by $e^{+} e^{-}$pairs? are the coronal parameters related to luminosity? what is the cause of the different X-ray properties of Seyfert galaxies and Quasars? could be answered in the near future.

\section{References}

Chen X., 1995, ApJ, 448, 903

Clavel J., et al., 1992, ApJ, 393, 113

Done C., Pounds K.A., Nandra K., Fabian A.C., 1995, MNRAS, 275, 417

Galeev A.A., Rosner R., Vaiana G.S., 1979, ApJ, 229, 318

Haardt F., 1993, ApJ, 413, 680

Haardt F., Maraschi L., 1991, ApJ, 380, L51

Haardt F., Maraschi L., 1993, ApJ, 413, 507

Haardt F., Maraschi L., Ghisellini G., 1994, ApJ, 432, L95

Haardt F., Maraschi L., Ghisellini G., 1996, ApJ, in press

Ionson J.A., Kuperus M., 1984 , ApJ, 284, 389

Johnson W.N., et al. 1993, A\&AS, 97, 21

Katz J.I., 1976, ApJ, 206, 910 
Kusunose M., Mineshige S., 1994, ApJ, 423, 600

Leighly K.M., Mushotzky R.F., Yaqoob T., Kunyeda H., Edelson R., 1996, ApJ, in press

Liang E.P.T., 1979, ApJ, 231, L111

Liang E.P.T., Price R.H., 1977, ApJ218, 247

Lightman A.P., Giacconi R., Tananbaum H., 1978, ApJ, 224,375

Madejski G.M., et al., 1995, ApJ, 438, 672

Mushotzky R.F., Done C., Pounds K.A., 1993, ARA\&A, 31, 717

Maisack M. et al., 1993, ApJ, 407, L61

Nakamura, Osaki, 1993, PASJ, 45, 775

Nandra K, Pounds K.A., 1994, MNRAS, 268, 405

Perola G.C., et al., 1936, ApJ, 306, 508

Pietrini P., Krolik J.H., 1995, ApJ, 447, 526

Pounds K.A., Nandra K., Stewart G.C., George I.M., Fabian A.C., 1990, Nat, 344, 132

Poutanen J., Svensson R., 1996, ApJ, 470, 249

Sanders D.B., Phinney E.S., Neuegebauer G., Soifer B.T., Matthews K., 1989, ApJ, 247, 29

Shakura N.I., Sunyaev R.A., 1973, A\&A, 24, 337

Shapiro S.L., Lightman A.P., Eardley D.M., 1976, apj, 204, 187

Shimura, Mineshige, Takahara, 1995, ApJ439, 74

Stern B.E., Poutanen J., Svensson R., Sikora M., Begelman M.C., 1995, ApJ, 449, L13

Sunyaev R.A., Titarchuk L.G., 1980, A\&A, 86, 121

Svensson R., 1994, ApJS, 92, 585

Svensson R., 1996, A\&A, in press

Svensson R., Zdziarski A., 1994, ApJ, 436, 599:

Titarchuk L.G., 1994, ApJ, 434, 313

Walter R., Fink H.H., 1993, A\&A, 274, 105

Witt H.J., Czerny B., Zycky P.T., 1996, MNRAS, submitted

Zdziarski A.A., Johnson W.N., Done C., Smith D., Mc Naron-Brown K., 1995, ApJ, 438, L63

Zycki P.T., Collin-Souffrin S., Czerny B., 1995, MNRAS, 277, 70

\section{Discussion}

U. Torkelsson: Assuming the corona consists of coronal loops. How far above the disc do these loops have to extend?

$L$. Maraschi: We assume the flat limit, that is that the down-scattered radiation, reprocessed in the disk, crosses the active region again without substantial losses. If the active region is at a height much larger than its dimension, it becomes "photon starved", i.e. the cooling is reduced and the comptonisation parameter is larger, producing flatter spectra. 